\title{
Produktpolitik
}

\section{Ökologische Perspektiven für eine bessere Chemiewirtschaft}

\author{
H. Friege \\ Dr. H. Friege, Umweltdezernent der Landeshauptstadt Düsseldorf, Stadtverwaltung Düsseldorf, Postfach 101120 , D-W-4000 Düsseldorf 1
}

Zusammenfassung. Qualitative und quantitative Probleme des Umsatzes von Stoffen zeigen sich in immer größerem Maße. Die gegen diese Auswirkungen industrieller Stoffwirtschaft gerichtete Chemiepolitik muß von der Chemieindustrie mit ihrer Schlüsselfunktion als stoffumwandelnde und Stoffströme lenkende Industrie positiv aufgenommen werden. Daraus resultiert eine Wandlung der Chemieindustrie vom bloßen Stoffproduzenten hin zum Dienstleister, der Stoffströme bewirtschaftet und technologische Problemlösungen anbietet, die über die reine Chemikalienproduktion hinausgehen. Eine ökologisch orientierte Umwandlung der Chemieindustrie kann durch entsprechende ökonornische Instrumente und der Beteiligung von Nutzern an stoffpolitischen Entscheidungen begünstigt werden.

\section{Grundlagen der Chemiepolitik}

Der Verbrauch an organischen Chemikalien hat sich zwischen dem Ende der 40er Jahre und dem Beginn der 80er Jahre etwa vervierzigfacht [1]. Der anthropogen bedingte Umsatz von Metallen wie Blei, Cadmium, Zink, Kupfer und Zinn beträgt heute ein Vielfaches der durch Erosion und Vulkanismus bedingten Emissionen [2]. Parallel zu dem allgemeinen Anstieg des Stoffumsatzes in der Chemieindustrie und verwandten Branchen sowie der qualitativen Veränderung durch eine Vielzahl neuer Verbindungen ist die Zunahme von Schadstoffrückständen in der Umwelt und beim Menschen selbst zu beobachten; daraus folgt ein steigendes Risiko unkontrollierter Reaktionen in der Umwelt und beim Menschen, die durch Einwirkung von Stoffen direkt oder indirekt verursacht werden.

Stark vereinfacht kann die globale Tendenz des heutigen Umganges mit Stoffen folgendermaßen zusammengefaßt werden: 1. mittelfristig ist mit einer erheblichen Verknappung von stofflichen bzw. energetischen Ressourcen zu rechnen, 2. kommt es zu einer weltweiten Verteilung von Schadstoffen [3].

Um diesen Entwicklungen entgegenzuwirken oder sie zu verlangsamen, wurden drei chemiepolitische Grundsätze formuliert [4]:

\section{Verlangsamung der Stoffströme:}

Ausgehend von den bereits eingetretenen Schäden in der Umwelt und der erheblichen Risikounschärfe im Hinblick auf weitere heute noch nicht erkennbare Schadwirkungen ist ein Minimierungsgebot für den Verbrauch von Stoffen einzuführen. Dies gilt nicht nur für alle schon als kritisch erkannten Substanzen, sondern auch für die Verwendung aller auf dem Markt befindlichen Stoffe.

\section{Schließung stofflicher Kreisläufe:}

Sofern Stoffe in geschlossenen oder zumindest gut kontrollierbaren offenen Systemen mit Rückführungsmechanismen eingesetzt werden, werfen sie deutlich geringere Risiken auf. Ein Recyclinggebot muß daher einer umweltverträglichen Stoffwirtschaft auf allen Ebenen von der Produktion über die Anwendung bis zur Abfallbehandlung Vorschub leisten. Dabei muß die Wiederverwendung eines Produktes Vorrang vor der bloßen Verwertung von Stoffen haben.

\section{3. Ökologisches Stoffdesign:}

Bei zahlreichen Anwendungsbereichen gibt es bestimmungsgemäß keine geschlossenen Systeme. Insbesondere für Stoffe, die in offenen Systemen verwendet werden, muß daher der Grundsatz des ökologischen Design gelten. Solche Chemikalien sollten so konzipiert sein, daß sie in natürliche Kreisläufe eingebaut werden können.

Das erste und zweite Prinzip wirken gegen die Exposition von Mensch und Natur durch Umweltchemikalien, wobei die Exposition im wesentlichen durch Produktionsmenge und Anwendungsmuster des jeweiligen Stoffes bestimmt wird. Das dritte Prinzip zielt auf die Verbesserung stofflicher Eigenschaften $(\rightarrow A b b$.). Dabei ist nicht nur der fragli-

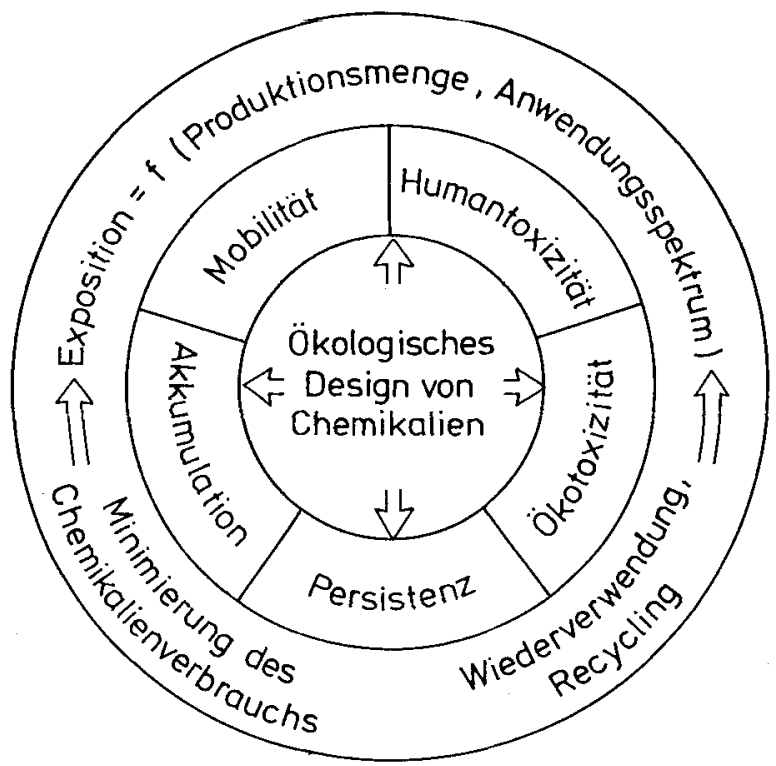

Abb.: Toxizität, Mobilität in Umweltmedien, Akkumulation, Persistenz sind kritische Eigenschaften von Stoffen, deren Auswirkungen sich bei Exposition zeigen. Das erste und zweite chemiepolitische Prinzip wirken der Exposition entgegen. Sofern der Stoff nicht in geschlossenen Systemen gehandhabt werden kann, muß das dritte Prinzip beachtet werden. 
che Stoff zu bewerten, sondern auch die jeweiligen Anwendungsfälle müssen betrachtet werden.

In den letzten Jahren hat sich die Erkenntnis durchgesetzt, daß die Umweltpolitik ihr Augenmerk auf Stoffe und Produkte richten muß, denn die eigentlichen Emissionen der chemischen Industrie sind nicht die im Zuge der Produktion entstehenden, mengenmäßig geringen Emissionen, sondern die Produkte selbst [5].

\section{Chemiepolitik und ökologisch-soziale Markt- wirtschaft}

Die genannten Grundregeln der Chemiepolitik sind in der Theorie mittlerweile relativ wenig umstritten [6]. Ihre Umsetzung wirft jedoch erhebliche Diskussionen auf, z.B.:

- die generelle Einschränkung des Stoffumsatzes [7],

- Kriterien der Risikobewertung [8], [9],

- Kriterien zur Bewertung des Nutzens [10].

Die Frage nach Kriterien für den Nutzen eines Stoffes ist eng verknüpft mit zwei grundsätzlich unterschiedlichen Philosophien der Chemieproduktion:

1. Insbesondere im Rahmen der Kuppelproduktion ${ }^{1}$ hat es sich eingebürgert, für Stoffe, z.B. Nebenprodukte, nach Anwendungsfeldern zu suchen.

2. Der umgekehrte Weg geht über die Definition einer Dienstleistung (z.B. Reinigung, Oberflächenschutz), für welche die technisch erforderlichen Chemikalien gesucht werden.

Anstatt durch eine Chemikalie kann die fragliche Dienstleistung aber oft auch auf anderem, z.B. mechanischem, Wege erbracht werden. Ein interessantes Beispiel bietet die Reinigung von Einrichtungen zur Autolackierung, die heute vielfach bereits mit Hochdruckwasser erfolgt, wohingegen früher $\mathrm{CKW}$ eingesetzt wurden.

Die Anwendung der chemiepolitischen Grundsätze fällt deutlich leichter, wenn man nicht den Nutzen eines Stoffs per se postuliert, sondern von Einsatzfeldern und Bedarf nach Dienstleistung (Chemiedienstleistung) ausgeht [6].

Die Frage der ökologischen Gestaltung einer künftigen Chemiewirtschaft ist auch eine strategische Frage der Chemiewirtschaft selbst, die sich zunehmend bewußt wird, daß der ökologische Aspekt eine zentrale Herausforderung der kommenden Jahrzehnte darstellt. Eine auch ökonomisch attraktive Perspektive ist für die Chemiewirtschaft nur in dem Ausmaße denkbar, wie sie mit ihren Produkten und Produktionsweisen auch ökologischen Kriterien gerecht wird [11]. Die Chemieindustrie hat bereits erkannt, daß es eine Gewichtsverschiebung vom additiven hin zum integrierten Umweltschutz geben muß [12].

Innerhalb einer Marktwirtschaft, die nicht nur sozialen, sondern auch ökologischen Fehlentwicklungen entgegensteuert, also einer ökologisch-sozialen Marktwirtschaft, ist es die wesentlichste Aufgabe, die Kräfte des Wettbewerbs, die Innovationsfähigkeit und die hohe Dynamik der Marktwirtschaft durch Schaffung von Rahmenbedingungen so zu

${ }^{1}$ Kuppelproduktion: Herstellung von nicht-erwünschten Produkten und Nebenprodukten neben dem erwünschten Reaktionsprodukt. nutzen, daß sie auf die Entwicklung einer stoff- und energieeffizienteren und umweltschonenderen Wirtschaftsweise wirken, ohne dabei soziale Probleme zu verschärfen [13].

Die Chemieindustrie hat eine Schlüsselstellung bei

- der Umwandlung von Stoffen,

- der Entwicklung und dem Angebot von Chemiedienstleistungen,

- der Lenkung von Stoffströmen.

Den Aufgaben der ökologisch-sozialen Marktwirtschaft kann die Chemieindustrie nur gerecht werden, wenn sie in allen drei Feldern die chemiepolitischen Grundsätze beachtet. Es spricht manches dafür, daß sich die Rahmenbedingungen der Chemiewirtschaft so ändern, daß sich eine umweltverträglichere Produktions- und Produktpolitik in Zukunft auszahlt. In der Tabelle sind einige Rahmenbedingungen aufgeführt, deren mittel- bzw. langfristige Tendenz vermutlich zu einer Kongruenz ökonomischer und ökologischer Ansprüche führen ( $\rightarrow$ Tabelle). Hinsichtlich der Verfügbarkeit und des Preises von Energie und Rohstoffen wird die langfristige Prognose gewagt, daß insbesondere die rohstofferzeugenden Länder in der Erzielung gerechter Preise erfolgreich sein werden. Zwischen 1990 und 2010 wird mit einer realen Verdoppelung der Energiepreise für Industrieabnehmer mit Ausnahme von Strom gerechnet [14]. Aufgrund der Nachfrage vor allem industrieller Kunden wird die Bedeutung von Spezialchemikalien zunehmen und die Nachfrage nach Massenchemikalien stagnieren bzw. abnehmen, zumindest, was den Verbrauch der hochindustrialisierten Länder betrifft [15].

Tabelle: Entwicklung von Rahmenbedingungen der Chemiewirtschaft

\begin{tabular}{lcc}
\hline Faktoren & mittelfristig & langfristig \\
\hline Energie & & \\
- Verfügbarkeit & + & - \\
- Preis & + & + \\
Rohstoffe & & \\
- Verfügbarkeit & + & - \\
- Preis & $?$ & + \\
Nachfrage nach Massenchemikalien & $(-)$ & - \\
Bedeutung von Bedarfsprofilen & + & + \\
Gebrauch kritischer Stoffea & - & - \\
Rücknahme von Stoffen/Produktena & + & + \\
Herstellerhaftung & + & $?$ \\
\hline
\end{tabular}

${ }^{a}$ Für technisch nicht verzichtbare kritische Stoffe bedarf es klar geregelter Verbleibnachweise und Rücknahmeverpflichtungen

Angesichts steigender Probleme in der Abfallwirtschaft wird die Rücknahme von Stoffen bzw. Produkten durch Hersteller oder Vertreiber zunehmen. Dies zeigt sich in Deutschland beispielsweise an der Verpackungsverordnung sowie an den schon angekündigten Rücknahmeverordnungen für Druckerzeugnisse, Computerschrott u.a.m. Rücknahmeverpflichtungen werden mittlerweile auch in anderen Industrieländern wie Japan eingeführt [16].

Industrielle wie private Verbraucher stellen zunehmend kritische Fragen hinsichtlich der Gesundheits- und Umweltverträglichkeit von Produkten. Einschränkungen der Chemikalienvielfalt, vor allem im Hinblick auf kritische Stoffe, sind insbesondere im Bereich des Arbeitsschutzes in Bran- 
chen zu erwarten, bei denen chemisch wenig vorgebildete Arbeitnehmer tätig sind, z.B im Bauhandwerk. Aber auch Anforderungen an

- eine Verbesserung der Innenraumluft,

- die Recyclingfähigkeit von Produkten allgemein,

- hautverträglichere Produkte im Bereich der Textilindustrie

stehen tendenziell der bislang fast ungebremst zunehmenden Stoff-Flut entgegen. In die gleiche Richtung wirkt auch die auf EG-Niveau angegangene Verschärfung der Herstellerhaftung mit der Tendenz zur verschuldensunabhängigen Produkthaftung.

Daraus ergibt sich insgesamt ein Trend zum Verkauf von Technologien anstatt nur von Stoffen, mithin ein Trend zur Chemiedienstleistung, wie auch die zunehmende Notwendigkeit für den industriellen Produzenten, die Distribution seiner Stoffe soweit wie möglich selbst zu kontrollieren.

Damit sollten folgende Strategien aufeinander abgestimmt werden:

1. Die Entwicklung weg von bloßer Massenproduktion geringer Wertschöpfung hin zum intelligenten Management großer Stoffströme,

2. die zunehmende Produktion ökologisch angepaßter Spezialitäten hoher Wertschöpfung,

3. die Bewirtschaftung von Stoffen von der Erzeugung bis zu ihrer Rücknahme bei Wiedereinbringung in den Produktionsprozeß im Sinne einer Vorwärtsintegration.

Die Chemieindustrie muß sich daher auch die Frage stellen, ob sie sich stärker als bisher in der Entsorgungswirtschaft engagieren will. Chemiedienstleistung kann auf lange Sicht auch heißen, daß Bedarfsgegenstände mit komplexer chemischer Zusammensetzung vom Benutzer nur gemietet werden, die darin enthaltenen Stoffe aber Eigentum des Produzenten bleiben, der sie nach Gebrauch zurückerhält. Ein solches Leasing langlebiger Güter und Produkte mit der Trennung von Nutzungs- und Verfügungseigentum sieht der Umweltsachverständigenrat als eine Möglichkeit zur Reduzierung von Abfallproblemen [17]. Der Produzent wird dann daran interessiert sein, diese Stoffe nicht einfach einer Oxidation (Verbrennung) oder Reduktion (Hydrierung) zu unterwerfen, sondern die wertvolle Komplexität der Molekülstrukturen soweit wie möglich zu erhalten. Ein erster Schritt wäre der Aufbau eines einheitlichen Rücknahmesystems für gefährliche Stoffe und deren Verpackungen. Henseling [13] sieht in der "lebenslangen Pflege“ an Produkten einschließlich ihrer Wiederverwendung ökonomische wie arbeitsmarktpolitische Chancen durch steigende Nachfrage nach entsprechenden Chemiedienstleistungen. Dies ist vor allem darin begründet, daß durch eine Verringerung neu zugeführter Rohstoffe die Qualifizierung im Bereich der Stoffumwandlungen, möglicherweise auch die Quantität der Stoffumwandlungen, zunimmt. Die Chemieindustrie muß die Chance wahrnehmen, ihr Know-how im Umgang mit gefährlichen Stoffen zu nutzen.

Eine klassische Vorstellung muß allerdings in Frage gestellt werden: Die stets steigende Vielfalt von Stoffen galt als Zeichen des Fortschritts. Um Strukturen für eine Wiederverwendung bzw. Wiederverwertung auf hohem Qualitätsniveau aufzubauen, bedarf es einer Reduktion der Differen- zierung bei Produkten hoher Distribution. Dies dürfte eine sinnvolle Lösung z.B. für den Einsatz von Kunststoffen auf dem Verpackungssektor sein, deren Sammlungs- und Wiederverwertungskosten z.Zt. mit etwa 2000 . - DM/t anzusetzen sind. Ähnliches gilt für die Bauwirtschaft.

\section{Beispiel: Chemie in der Bauwirtschaft}

In Bauwerken sind die quantitativ bedeutendsten Mengen an Abfällen "zwischengelagert": sie kommen nach Ende der Nutzungsdauer auf die Abfallwirtschaft zu. Die Bewältigung dieses Depots ist nicht nur ein quantitatives Problem, sondern auch zunehmend ein qualitatives.

Je mehr der Abbruch eines Hauses einem Rückbau ähnelt, und je mehr schadstoffbelastete Materialien dabei nicht verwertet, sondern als Abfall entsorgt werden müssen, desto teurer wird dies für den Bauherrn, der daraus auch Lehren für die künftige Verwertung und Verwendung von Baustoffen und Bauchemikalien ziehen wird. Die Kosten für das Recycling von Abbruchmaterialien und Baustellenabfällen hängen im wesentlichen zusammen mit

- dem Schadstoffinventar des Baus,

- der Abtrennbarkeit von Schadstoffen über den Ausbau definierter Bauteile,

- der Verfügbarkeit eines Marktes für die Recyclingprodukte.

Dabei erbringt das Recycling bei zunehmenden organischen Anteilen im Bauschutt nur Material, das sich nicht für eine höherwertige Nutzung eignet. Es ist aus Sicht des Autors sehr empfehlenswert, diese Zusammenhänge in einer zukunftsorientierten Produktpolitik von Chemieindustrie und Bauwirtschaft aufzuarbeiten.

\section{Steuerungsinstrumente für eine ökologische Per- spektive der Chemiewirtschaft}

Die klassische Umweltpolitik ist vor allem auf den Schutz der Umweltmedien gerichtet. Sie arbeitet im wesentlichen mit einem ordnungsrechtlichen Instrumentarium, mit dem die Konzentration, in letzter Zeit auch zunehmend die Fracht von Schadstoffen, begrenzt werden.

Chemiepolitik ist dagegen auf die Lenkung von Stoffströmen auszurichten. Dabei geht es zum einen um die Begrenzung der Anwendung gefährlicher Stoffe, z.B. durch Einengung des Anwenderkreises oder im Extremfall auch durch ein Produktions- und Anwendungsverbot. Ferner muß Chemiepolitik die Schließung von Schadstoff- und Wertstoffkreisläufen zum Ziel haben, wobei Schadstoffe aus Wertstoffkreisläufen ausgeschleust und sicher entsorgt oder in den Produktionsprozeß zurückgeführt werden müssen. Ein typisches Beispiel hierfür ist die Entfrachtung des Wertstoffes Klärschlamm von Schadstoffen durch Abwasservorreinigung.

Im Gegensatz zur klassischen Umweltpolitik, die mit relativ schlecht überschaubaren Abluft-, Abwasser- und Abfallströmen fertig werden muß, zielt die Chemiepolitik auf vergleichsweise gut definierte und damit kontrollierbare 
Stoffströme. Stoffströme können auf verschiedenen Stufen (Rohstoffe, Produkte, Abfälle) einer Steuerung durch Einsatz ökonomischer Instrumente wie Steuern oder Abgaben unterworfen werden. Chemiepolitik ist somit ein Bereich, in dem sich der Einsatz ökonomischer Instrumente geradezu anbietet. Damit könnte gleichzeitig die Gefahr vermieden werden, durch ordnungsrechtliche Überreglementierung an anderer Stelle dringend benötigte Arbeitskraft zu binden, so daß letztlich Vollzugsdefizite vermieden werden könnten [18].

Zur Steuerung von Stoffströmen kommen beispielsweise Abgaben auf besonders toxische, aber $\mathrm{z} . \mathrm{Zt}$. noch unverzichtbare Stoffe in Frage. Unter Bezugnahme auf die chemiepolitischen Grundprinzipien wurde beispielsweise eine Steuer vorgeschlagen, die sich an der in Verkehr gebrachten Menge einer Chemikalie, ihrer Toxizität und ihrer Persistenz orientiert und die um so geringer wäre, je stärker dieser Stoff durch Schließung des Stoffkreislaufs in die Chemieindustrie zurückgeführt würde [19].

Gerechtere, d.h. höhere Weltmarktpreise für Rohstoffe hätten durch die Anreizwirkung zur Entwicklung stoffeffizienterer Technologien positive ökologische Auswirkungen. Sie sind daher nicht nur entwicklungspolitisch, sondern auch umweltpolitisch wünschenswert.

Die Verschärfung von Produkthaftungsregeln ist ein wenn auch nicht auf einzelne Stoffe zielendes - Instrument der Chemiepolitik, das tendenziell die Verwendung weniger kritischer Stoffe fördert und gleichzeitig dem schnellen Produktwechsel entgegensteht, weil verschärfte Produkthaftung den $F+E$-Aufwand erhöht.

Eine erste und unabdingbare Voraussetzung für die Bewirtschaftung von Stoffströmen ist die Erstellung von Stoffbilanzen, wie sie nunmehr in Form der Chlorbilanz [20] des Umweltbundesamtes vorliegt. Die Chemieindustrie tut sich mit Informationen über die von ihr verursachten Stoffströme noch schwer. Die neue Altstoffverordnung der EG, zunehmender Informationsbedarf von industriellen Kunden der Chemieindustrie sowie von Privatverbrauchern und der Trend zum Erlaß von Rücknahmeverpflichtungen werden die Hersteller zu einer stärkeren Offenlegung von qualitativen und quantitativen Stoffdaten zwingen. Eine freiwillige Offenlegung von Daten über interne Stoffströme, Stoffinput und Stoffoutput von Produkten wäre der erste Schritt zu einer ökologischen Unternehmenskultur.

\section{Ausblick}

Globale und lokale Probleme beim Umgang mit Chemikalien, Stoffen und Produkten zwingen zum Umdenken. Stoffströme müssen sparsam und umweltverträglich bewirtschaftet werden, um aus ihnen größtmöglichen Nutzen bei Minimierung der damit verbundenen Schäden oder Risiken zu ziehen. Ein großer Teil des dafür erforderlichen Rüstzeugs steht zur Verfügung: die Erkenntnisse über grundlegende Verhaltensmuster von Stoffen in Umweltmedien und Umweltkompartimenten, toxikologische Grunddaten und ökonomische Instrumente, die auf anderen Feldern erprobt wurden. Es ist wichtig, die Begrenztheit unseres Wissens zu akzeptieren. Die Synthesechemie wird immer weiter sein als die Kenntnis der Stoffwirkungen dies ist eine entscheidende Erkenntnis für den verantwortlichen Umgang mit chemischen Stoffen.

Dazu ist ein Umdenken des Chemieunternehmers erforderlich:

- vom bloßen Stoffproduzenten hin zum Dienstleister, dessen Stärke in der Kenntnis der technischen und ökochemischen Stoffe liegt,

- vom Hersteller hin zum Stoffstrombewirtschafter, dessen Verantwortung den Produktlebensweg umfaßt.

Die vom Bundestag im März 1992 eingesetzte EnquêteKommission „Schutz des Menschen und der Umwelt - Bewertungskriterien und Perspektiven für umweltverträgliche Kreisläufe in der Industriegesellschaft" wird hierzu Vorarbeiten leisten.

\section{Danksagung}

Für hilfreiche und kritische Diskussionen danke ich Dr. Karl Otto Henseling, Dr. Wolfgang Linden, Dr. Inge Bantz, Dr. Werner GörTZ und Prof. Dr. Eberhard WeISE.

\section{Literatur}

[1] Editorial: Exchange of information and chemicals in international trade: Time for action, IRPTC-Bulletin 9, 1 - 2 (1988)

[2] H. Friege; U. Kost; F. Claus (Hrsg.): Die tückische Hypothek - Chemiepolitik für Schwermetalle, Karlsruhe 1985

[3] M. HELD (Hrsg.): Chemiepolitik: Gespräch über eine neue Kontroverse, Weinheim 1988

[4] H. Friege; F. Claus (Hrsg.): Chemie - für wen? Reinbek 1988

[5] E. WEISE: Grundsätzliche Überlegungen zur Verbreitung und Verbleib von Gebrauchsstoffen. In [6], 55-64

[6] M. Held (Hrsg.): Leitbilder der Chemiepolitik. Frankfurt, New York 1991

[7] B. BROECKER: Expositionsanalyse in der Schadstoffbewertung. Nachr. Chem. Tech. Lab. $101-104$ (1990)

[8] J. MEERKAMP v. EMBDEN: Grundlagen einer umweltverträglichen Stoffwirtschaft. In [10], $109-122$

[9] K. KümmereR: Risiko und Nutzen, In [10], 23-44

[10] Umweltdezernat Frankfurt/M. (Hrsg.): Zweite Frankfurter Studie zur Förderung chemiepolitischer Verständigungsprozesse, Teil II, Frankfurt 1992

[11] A. Ueberhorst; R. de MaN: Zweite Frankfurter Studie zur Förderung chemiepolitischer Verständigungsprozesse, Teil I, $53 \mathrm{ff}$, Frankfurt 1992

[12] K. HolubeK: Perspektiven der Chemieproduktion am Beispiel Chlor. In: Chemie im Dialog (Hrsg.: VCI), 1991

[13] K. O. Henseling: Zukunft der Chemie in der ökologischsozialen Marktwirtschaft. In [10], 93-98

[14] Enquête-Kommission „Vorsorge zum Schutz der Erdatmosphäre“ des Dt. Bundestags (Hrsg): Energie und Klima, Band 1, 77, 104, Bonn/Karlsruhe, 1990

[15] T. Sommerlatte; K. Tiby: Spielregeln der Zukunft. Chem. Ind. $50-52(1988)$

[16] H. FRIEGE: Abfallwirtschaft in Japan, Müll und Abfall 165 - 171 (1992)

[17] Der Rat der Sachverständigen für Umweltfragen: Sondergutachten Abfallwirtschaft, $\mathrm{Tz}$.

[18] H. FrIEGE; K. O. HeNSELING: Regelungsmängel und Vollzugsdefizite. In [10], $81-88$

[19] E. Holtmeier; H. Friege: In [4], 213-221

[20] Umweltbundesamt (Hrsg.): Handbuch Chlorchemie I, UBATexte 55/91, Berlin 1991 